\title{
Leiomioma benigno metastizante: caso clínico con nódulos pulmonares e histerectomía 4 años antes por leiomiomas uterinos
}

\author{
Francisco Mucientes H. ${ }^{1}$, Carlos Robalino S. ${ }^{1}$, Rodrigo Klaassen P. ${ }^{1}$, Emilio \\ Alarcón C. 2 , Fernando Chuecas S. 3 \\ ${ }^{1}$ Sección Patología, ${ }^{2}$ Departamento de Cirugía, Facultad de Medicina, Universidad de Concepción. ${ }^{3}$ Facultad de \\ Ciencias Médicas, Universidad Católica de la Santísima Concepción. 8ª Región, Chile.
}

\section{RESUMEN}

Se presenta un caso de leiomioma pulmonar metastizante en una mujer de 48 años. El estudio histológico reveló múltiples nódulos pulmonares de musculatura lisa de aspecto benigno. El seguimiento a 8 años mostró una paciente en buenas condiciones generales sin síntomas respiratorios. La historia clínica reveló antecedentes de histerectomía cuatro años antes debido a múltiples leiomiomas. Se hace una revisión clínico patológica de los tumores musculares lisos uterinos en sus formas clásicas y en sus presentaciones inusuales.

\section{PALABRAS CLAVE: Leiomioma metastizante benigno, mioma}

\section{SUMMARY}

A case of pulmonary benign metastasizing leiomyoma in a 48 years old female is presented. The histological study showed pulmonary nodules formed by smooth muscle cell of benign appearance. The 8 years follow up revealed a patient in a good fashion without respiratory symptoms. There was a 4 years history of previous hysterectomy due to multiple uterine leiomyomas. A clinico pathological review of the classical and unusual forms of smooth muscle tumors of the uterus is also presented.

\section{KEY WORDS: Benign metastasizing leiomyoma, leiomyoma}

\section{INTRODUCCIÓN}

Existe una gran variedad de tumores musculares lisos del útero que en la mayoría de los casos pueden ser clasificados sin grandes dificultades en entidades de comportamiento benigno o maligno. Sin embargo, permanece una serie de tumores musculares donde esta diferenciación no es posi- ble, debiendo ser clasificados en un grupo de comportamiento biológico incierto. Además se han descrito una serie de neoplasias o lesiones musculares lisas raras, tanto en su presentación clínica, como en su origen histogenético y que generan dificultades diagnósticas y terapéuticas.

A fin de situar el caso de Leiomioma Pulmonar Metastizante que se presenta dentro del espectro 
de los tumores musculares lisos uterinos, se hará una revisión de estas neoplasias incluyendo criterios histopatológicos diagnósticos actuales.

Aspectos clínicos. La mayoría de los leiomiomas del útero se presentan en mujeres de edad fértil y usualmente como múltiples nódulos bien delimitados. A diferencia, el leiomiosarcoma se ve en mujeres más añosas y con tumores usualmente únicos e infiltrantes, con una media de $10 \mathrm{~cm}$ de diámetro. La presentación clínica para ambas entidades es muy semejante por lo que la detección preoperatoria es difícil. Sin embargo, se debe sospechar malignidad cuando aparecen ciertos hallazgos clínicos como crecimiento tumoral en mujer menopáusica sin terapia de reemplazo hormonal, rotura, hemoperitoneo, extensión extrauterina o metástasis $(1,2,3)$.

Parámetros histológicos diagnósticos. El diagnóstico histológico para los diferentes neoplasmas de musculatura lisa se establece aplicando un conjunto de parámetros que incluyen presencia de necrosis tumoral (necrosis mala), atipia moderada a intensa difusa, y conteo de mitosis de $\geq 10$ por 10 campos microscópicos de aumento mayor. Para la forma histológica fusocelular (tipo usual) se ha propuesto que con dos de estos criterios histológicos se puede distinguir con seguridad entre leiomioma y leiomiosarcoma $(4,5)$. Para otras variantes histológicas los criterios cambian, así, para los tumores musculares lisos mixoídeos se considera cualquiera de los siguientes parámetros: atipia moderada a intensa, $\geq 2$ mitosis por 10 campos mayores 0 necrosis tumoral. En cambio, para la variante epitelioide se considera: atipia moderada a intensa y $\geq 5$ mitosis por 10 campos mayores, o necrosis tumoral $y \geq 5$ mitosis por 10 campos mayores (2).

Excepciones a parámetros histológicos diagnósticos. Para cada uno de estos parámetros histológicos tomados en forma independiente existen excepciones, las que incluso en algunos casos, definen entidades particulares con poca o no recurrencia, entre las que se encuentran: leiomioma con núcleos bizarros o atípicos que tienen usualmente distribución focal, mitosis aisladas o ausentes y sin necrosis tumoral; leiomioma necrótico o apopléctico (presencia de necrosis tipo infarto o hialina (necrosis buena), sin atipia ni mitosis significativas); leiomioma celular y altamente celular (significativamente más celular que el endometrio adyacente o alta densidad celular respectivamente, sin atipia significativa ni necrosis tumoral y mitosis en rangos de 1 a 4 mitosis por 10 campos mayores); leiomioma mitóticamente activo ( $\geq 5$ hasta 15 mitosis por 10 campos mayores) $(2,6)$.

Tumores musculares de potencial maligno incierto. A pesar de la aplicación de estos parámetros his- tológicos, quedan algunos casos de tumores musculares inclasificables bajo los criterios actuales dentro de los grupos categóricamente benignos o malignos. En este caso la neoplasia debe ser catalogada como tumor de musculatura lisa de potencial maligno incierto con su sigla en inglés STUMP.

A pesar de la histología algo preocupante de estos tumores, la mayoría se comporta en forma benigna aunque algunos pudieran recurrir en el tiempo en forma de STUMP o de leiomiosarcoma en cifras promedio de $7-10 \%(2,5,7)$. El término de leiomioma atípico es controversial y es usado por algunos como un eufemismo para neoplasias musculares tipo STUMP, mientras que otros lo usan como sinónimo de leiomioma con núcleos bizarros. Esta controversia se produce en parte por la aceptación de los criterios histológicos de atipia. Para este grupo de neoplasias musculares de pronóstico incierto hace necesario un seguimiento a largo plazo con exámenes clínicos y de imagen. La terapia varía dependiendo si la paciente ha completado la paridad e incluye histerectomía, y en casos de recurrencia el tratamiento es la resección quirúrgica y la terapia adyuvante con análogos hormonales y quimioradiación $(2,5)$.

Tumores musculares de localización excepcional. Se han descrito una serie de entidades raras de tumores musculares lisos que no cumplen los criterios para el diagnóstico de leiomiosarcoma pero que muestran una localización inusual, lo que sugiere malignidad y entre los que se encuentran:

a. Leiomioma disecante. Se trata de un leiomioma con patrón de crecimiento inusual consistente en permeación a través del miometrio, extensión al ligamento ancho, a veces con compromiso de la pelvis, y con marcada congestión vascular, dando un aspecto de cotiledones placentarios. Cuando el tumor aparece en la subserosa y compromete el ligamento ancho, sin el componente miometrial disecante, se lo puede llamar leiomioma citiledonoide o cotiledónico (2).

b. Leiomiomatosis peritoneal diseminada. Se presenta como múltiples nódulos de musculatura lisa sin atipia y localizados en el epiplón y peritoneo. Puede asociarse a leiomiomas uterinos (8).

c. Leiomiomatosis intravenosa. Corresponde a una proliferación intravenosa de musculatura lisa, histológicamente benigna y fuera de los confines de un leiomioma uterino, o a veces en ausencia de este tumor. El hallazgo quirúrgico corresponde a tapones de células musculares lisas en cualquiera de sus variantes benignas, que llenan las venas pélvicas ocupando desde el ligamento ancho a la vagina $(9,10)$.

d. Linfangioleiomiomatosis. Se ve proliferación de 
nódulos de células mioides fusadas o células claras en canales linfáticos con compromiso de pulmones y ganglios linfáticos, positivas a la inmuno tinción HMB45 y a veces asociada a esclerosis tuberosa (11).

e. Leiomioma benigno metastizante. Muestra nódulos múltiples y bilaterales de musculatura lisa, histológicamente benigna, localizados en los pulmones aunque también puede encontrarse en ganglios linfáticos o cavidad abdominal $(12,13)$. Esta lesión parece originarse o asociarse con leiomiomas uterinos resecados muchos años antes de la enfermedad extrauterina $(2,14)$. El curso clínico es lento y benigno, salvo complicaciones por crecimiento y compresión de estructuras vecinas.

\section{Caso clínico}

Mujer de 48 años en excelentes condiciones generales, consulta en mayo de 2002 por dolor abdominal bajo. El examen ginecológico y estudio ecográfico mostró el anexo derecho de localización posterior con ovario aumentado de tamaño, sólido quístico. Los niveles de $\mathrm{Ca} 125$ fueron de $1587 \mathrm{U} /$ $\mathrm{ml}$ (normal $<40 \mathrm{U} / \mathrm{ml}$ ). El estudio radiológico reveló múltiples nódulos pulmonares bilaterales consistentes con metástasis. Se realiza laparotomía exploradora donde se encuentra focos de endometriosis quística en el ovario y peritoneo pélvico, que son resecados y confirmados por biopsia. No se demuestra neoplasia intraabdominal. Postoperatorio sin complicaciones, con niveles de $\mathrm{Ca} 125$ que disminuyen a $171 \mathrm{U} / \mathrm{ml}$. Entre los antecedentes destaca la historia de histerectomía total en 1998 y salpingoooforectomía izquierda. La biopsia del útero reveló múltiples nódulos miomatosos, sin presencia de atipia, necrosis tumoral o mitosis. El ovario y la trompa no mostraban lesiones.

Se decide ampliar estudio para definir naturaleza de los nódulos pulmonares. Se realiza TAC de tórax (Figura 1) y posterior biopsia de uno de los nódulos pulmonares periféricos bajo visión directa por toracoscopia. La biopsia pulmonar consistió en una cuña tejido de $2,2 \times 0,5 \times 0,4 \mathrm{~cm}$ con dos nódulos blanquecinos, uno subpleural de $0,5 \mathrm{~cm}$ y otro más profundo de $0,2 \mathrm{~cm}$. A la histología, los dos nódulos estaban formados por una proliferación de células fusadas con atrapamiento de epitelio pulmonar bronquial (Figura 2). Las células revelaron núcleos fusados en cigarro y formación de fascículos entrecruzados. No se observó mitosis, necrosis, ni atipia (Figura 3). Las tinciones de inmunohistoquímica mostraron fuerte reactividad para marcadores

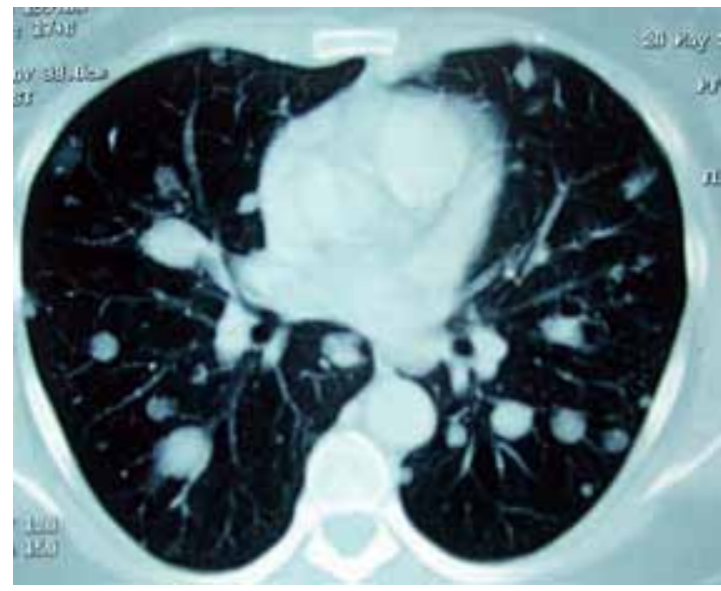

Figura 1. Vista radiológica de nódulos pulmonares múltiples bilaterales (mayo de 2002).

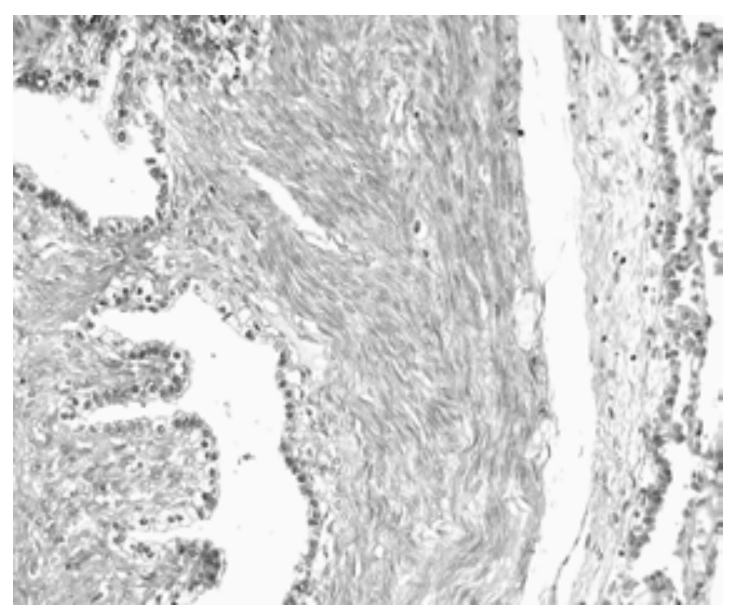

Figura 2. Vista microscópica a bajo aumento, tinción H\&E, de un nódulo pulmonar con atrapamiento epitelial bronquial y a la derecha rodeado por parénquima alveolar.

musculares como actina y desmina. La tinción para citoqueratina resultó negativa en células fusadas y positiva en el componente epitelial atrapado. El antígeno de proliferación Ki-67 indicó menos del 1\% de núcleos positivos. Los receptores estrogénicos y Bcl-2 fueron negativos. El seguimiento muestra un último control en diciembre de 2011, con una paciente en buen estado general, activa, sin disnea y con nódulos pulmonares estables. 


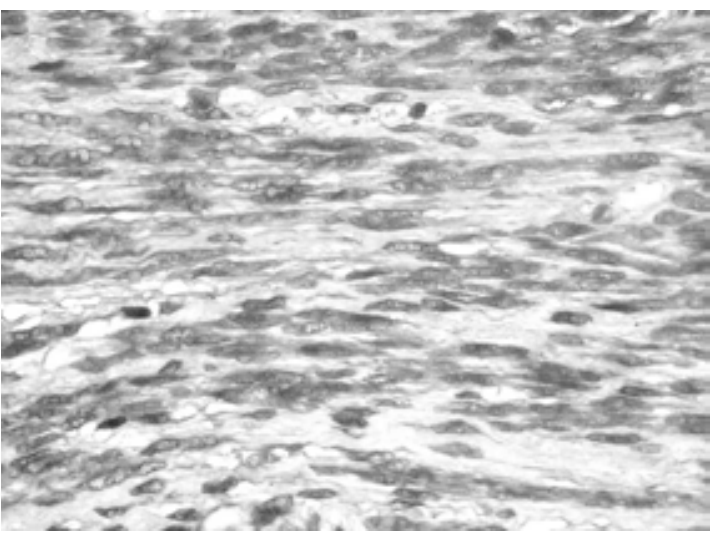

Figura 3. Vista microscópica a gran aumento, tinción H\&E, de un nódulo pulmonar con células musculares lisas de aspecto benigno, sin atipias, necrosis ni mitosis.

\section{DISCUSIÓN}

Diagnóstico. El término de leiomioma benigno metastizante es un mal nombre que revela la incertidumbre clínico patológica, y falta de una histogénesis categórica para esta extraña lesión. El diagnóstico debe realizarse sólo cuando se ha excluido un leiomiosarcoma uterino o extrauterino, y que cualquier leiomioma uterino preexistente haya sido muestreado y debidamente estudiado para descartar histología de malignidad (2). Esto no es fácil de realizar, debido a que el tumor uterino suele haber sido resecado muchos años antes y usualmente no se encuentra material disponible para revisión histológica. Como se espera, esta entidad es clínicamente mal interpretada como metástasis de leiomiosarcoma uterino. Sin embargo, lo clásico para el leiomioma benigno metastizante es la asociación con tumores uterinos que al microscopio califican como leiomiomas o formado por células musculares de características histológicas benignas. A diferencia, el leiomiosarcoma uterino que revela bordes infiltrativos, necrosis tumoral, y que bajo el microscopio cumple con los criterios histológicos actuales de malignidad, que incluye una combinación de necrosis tumoral, alto conteo de mitosis y atipia celular significativa $(2,4)$. Estos criterios no forman parte de los tumores uterinos descritos en asociación con el leiomioma benigno metastizante.

La resección del tumor, en este caso pulmonar, con el estudio histológico correspondiente es crucial para establecer el diagnóstico de leiomioma benigno metastizante, ya que la contrapartida maligna, es decir el leiomiosarcoma puede mostrar el mismo aspecto al examen clínico y al estudio por imagen (14).

Curso clínico y tratamiento. El curso clínico es muy lento, y clásicamente señalado como benigno, pero los tumores pulmonares pueden continuar creciendo y producir falla respiratoria y muerte (15). Por esta razón, algunos han propuesto que estas neoplasias deberían al menos ser llamadas como tumor muscular liso de pronóstico incierto o STUMP (16). No hay un tratamiento establecido para esta lesión debido a la rareza de los casos. La resección quirúrgica completa de los nódulos asociada con terapia hormonal se relaciona con sobrevida prolongada. En un caso excepcional de tumores pulmonares múltiples, se ha usado tratamiento con argón-plasma para resecar uno de los nódulos que hacía obstrucción endobronquial (17). Como son neoplasias hormono dependientes se deben suspender los anticonceptivos orales o la terapia de reemplazo hormonal. Se ha reportado reducción de los nódulos con la terapia de supresión ovárica, incluyendo inhibidores de aromatasa (18-20). El uso de progesterona puede servir para acelerar la regresión de los nódulos y evitar la cirugía radical (21). En casos de enfermedad progresiva irresecable, o cuando el tumor es negativo para receptores de estrógeno y progesterona, se ha indicado quimioterapia sistémica con dexorubicina y dacarbazina $(22,23)$.

Patogénesis. La patogénesis es incierta y para explicar cómo un tumor uterino de histología benigna pueda diseminarse a diferentes sectores u órganos, en especial a los pulmones, se han propuesto una serie de hipótesis explicativas: 1 . diseminación linfovascular de un leiomioma uterino benigno, debido a disrupción mecánica. 2. metástasis de un leiomiosarcoma de bajo grado que fue mal muestreado, o si fue bien muestreado no se revela como tal a la histología. 3. proliferación de musculatura lisa hormonalmente inducida. 4. neoplasia muscular lisa de origen multifocal.

La evidencia actual apunta mejor a una diseminación linfática o vascular a partir de leiomioma del útero. La existencia de tumores híbridos como leiomiomas uterinos con invasión vascular o leiomiomatosis intravascular pélvica con nódulos pulmonares de leiomioma benigno metastizante, sugieren que el compromiso pulmonar se produce vía leiomiomatosis intravenosa $(10,24-27)$. Se ha propuesto recientemente un mecanismo similar al de la endometriosis (28). Desde el punto de vista molecular se ha encontrado patrones idénticos de inactivación alélica del receptor androgénico e inactivación del cromosoma X, entre los nódulos pulmonares y uterinos, demostrando que ambas lesiones son clonales $(16,29)$. Algunos estudios han encontrado un perfil citogené- 
tico distintivo en el leiomioma benigno metastizante y en un grupo pequeño de leiomiomas uterinos, perfil ausente en el resto de los tumores musculares benignos o malignos estudiados, lo que apoya que el origen de la lesión pulmonar es en un grupo muy especial de leiomiomas del útero (30). Estudios moleculares recientes indican que se trataría de lesiones musculares benignas ya que no tienen el micro RNA MIR-221 que usualmente se encuentra en una serie de cánceres (31).

\section{REFERENCIAS}

1. D'Angelo E, Prat J. Uterine Sarcomas: A review. Gynecol Oncol 2010;16:131-9.

2. Ip PPC, Tse KY, Tam KF. Uterine smooth muscle tumors other than the ordinary leiomyomas and leiomyosarcomas: a review of selected variants with emphasis on recent advances and inusual mophology that may cause concern for malignancy. Adv Anat Pathol 2010;17:91-112.

3. Jones MW, Norris HJ. Clinicopathologic study of 28 uterine leiomyosarcomas with metastasis. Int J Gynecol Pathol 1995;14:243-9.

4. Bell SW, Kempson RL, Hendrickson MR. Problematic uterine smooth muscle neoplasms. A clinicopathologic study of 213 cases. Am J Surg Pathol 1994;18:535-58.

5. Ip PP, Cheung AN. Pathology of uterine leiomyosarcomas and smooth muscle tumors of uncertain malignant potential. Best Pract Res Clin Obstet Gynecol 2011;25:691-704.

6. Payson RA, Hart WR. Mitotically active leiomyomas of the uterus. Am J Clin Pathol 1992;97:14-20.

7. Deodhar KK, Goyal P, Rekhi B, et al. Uterine smooth muscle tumors of uncertain malignant potential and atypical leiomyoma: a morphological study of these grey zone with clinical correlation. Indian J Pathol Microbiol 2011;54:706-11.

8. Montero JC, Iturain N, Urrea D, Missad C. Leiomiomatosis peritoneal diseminada y embarazo. Rev Chil Obstet Ginecol 2008;73:134-7.

9. Canzonieri V, D’Amore ES, Bartoloni G, et al. Leiomyomatosis with vascular invasion. A unified pathogenesis regarding leiomyoma wih vascular microinvasion, benign metastasizing leiomyoma and intravenous leiomyomatosis. Virchows Arch 1994;425:541-5.

10. Clement PB, Young RH, Scully RE. Intravenous leiomyiomatosis of the uterus. A clinicopathologic análisis of 16 cases with unusual histological features. Am J Surg Pathol 1988;12:932-45.

11. Han JM, Lee KH, Kim SJ, et al. A case of lymphangioleiomyomatosis originated in the pelvic cavity. J Gynecol Oncol 2008;19:195-8.

12. Goto T, Maeshima A, Akanabe K, et al. Benign metastasizing leiomyoma of the lung. Ann Thorac Cardiovasc Surg 2012;18:121-4.

13. Rege AS, Snyder JA, Scott WJ. Benign metastasizing leiomyoma: a rare cause of multiple pulmonary nodules. Ann Thorac Surg 2012;93:e149-51.

14. Ito $\mathrm{T}$, Kawasaki N, Kinoshita M, et al. Benign metas- tasizing leiomyoma: report of a case. Kyobu Geka 2012;65:513-5.

15. Kayser K, Zink S, Schneider T, et al. Benign metastasizing leiomyoma of the uterus: documentation of clinical, immunohistochemical and lectin-histochemical data of ten cases. Virchows Arch 2000;437:284-92.

16. Patton KT, Cheng L, Papavero V, et al. Benign metastasizing leiomyoma: clonality, telomere length and clinipathologic analysis. Mod Pathol 2006;19:130-40.

17. Bugalho A, Oliveira A, Sernedo J, et al. Argon-plasma treatment in benign metastasizing leiomyoma of the lung: a case report. Rev Port Pneumol 2010;16:921-3.

18. Taveira-Da Silva AM, Alford CE, Levens ED, Moss J. Favorable response to antigonadal therapy for a benign metastasizing leiomyoma. Obstet Gynecol 2012;119:438-42.

19. Beck M, Biswas B, D'Souza A, et al. Benign metastasizing leiomyoma after hysterectomy and bilateral salpingo-oophorectomy. Hong Kong Med J 2012;18:153-5.

20. Rivera JA, Christopoulos S, Small D, et al. Hormonal manipulation of benign metastasizing leiomyoma: a report of two cases and review of the literature. $\mathrm{J}$ Clin Endocrinol Metab 2004;89:3183-8.

21. Wentlig GK,Sevin BU,Geiger XJ, et al. Benign metastasizing leiomyoma responsive to megestrol: a case report and review of the literature. Int J Gynecol Cancer 2005;15:1213-7.

22. Lin YC, Wei LH,Shun CT, et al. Disseminated peritoneal leiomyomatosis to systemic chemotherapy. Oncology 2009;76:55-8.

23. Jiang GQ, Gao YN, Gao M, et al. Benign metastasizing leiomyoma: report of two cases and literature review. Chin Med J 2010;123:3367-71.

24. Bodner-Adler B, Bartl M, Wagner G. Intravenous leiomyomatosis of the uterus with pulmonary metastases or a case with benign metastasizing leiomyoma? Anticancer Res 2009;29:495-6.

25. Lee HJ, Choi J, Kim KR. Pulmonary benign metastasizing leiomyoma associated with intravenous leiomyomatosis of the uterus: clinical behaviour and genomic changes supporting a transportation theory. Int J Gynecol Pathol 2008;27:340-5.

26. Arif S, Genesan R, Spooner D. Intravascular leiomyomatosis and benign metastasizin leiomyoma: an unusual case. Int J Gynecol Cancer 2006;16:1448-50.

27. Koh DM, BurnPR, King DM. Benign metastasizing leiomyoma with intravascular leiomyomatosis $\mathrm{Br} \mathrm{J} \mathrm{Ra}$ diol 2002;73:435-7.

28. Owonuga AO, Shavell VI, Imudia AN, et al. Pathogenesis of benign metastasizing leiomyoma: a review. Obstet Gynecol Surv 2010;65:189-95.

29. Tietze L, Gunther K, Horbe A, et al: Benign metastasizing leiomyoma: a cytogenetically balanced but clonal disease. Hum Pathol 2000;31:126-8.

30. Nucci MR, Drapkin R, Dal Cin P, et al. Distintive cytogenetic profile in benign metastasizin leiomyoma: pathogenetic implications. Am J Surg Pathol 2007;31:737-43.

31. Nuovo GJ, Schmittgen TD. Benign metastasizing leiomyoma of the lung: clinicopathologic, immunohistochemical and micro-RNA analysis. Diag Mol Pathol 2008;17:145-50. 